\title{
Realization of the First Aplanatic Transmission Electron Microscope
}

I. Maßmann*, S. Uhlemann*, H. Müller*, P. Hartel*, J. Zach*, M. Haider*, Y. Taniguchi**, D. Hoyle***, and R. Herring****.

*Corrected Electron Optical Systems GmbH, Englerstr. 28, D-69126 Heidelberg, Germany.

**Hitachi High-Technology Cooperation, 882, Ichige, Hitachinaka, Ibaraki, 312-8504 Japan.

***Hitachi High-Technology Ltd., 89 Galaxy Blvd. Suite 14, Toronto, Ontario, Canada, M9W 6A4. ****University of Victoria, P.O. Box 3055, Victoria, B.C., V8W 3P6 Canada.

During the last decade aberration correctors have become well-accepted tools in high-resolution transmission electron microscopy [1]. These correctors compensate for the spherical aberration $\mathrm{C}_{\mathrm{s}}$ of the imaging system. Also the simultaneous correction of the chromatic aberration $\mathrm{C}_{c}$ has been demonstrated successfully at an energy range from $80 \mathrm{kV}$ to $300 \mathrm{kV}$ [2]. For instruments with considerably improved information limit recently the off-axial aberrations have attracted more attention since these aberrations can limit the high-resolution field of view. Parasitic second-order off-axial astigmatism and third-order azimuthal off-axial coma $\mathrm{B}_{3}=\mathrm{B}_{2 \bar{\gamma}}$ are most critical among these generalized coma aberrations [1].

To fulfill the need for high-resolution imaging with large CCD detectors with 4kx4k pixels and more we have proposed [1] and developed [3] a novel hexapole-type $\mathrm{C}_{\mathrm{s}} / \mathrm{B}_{3}$-corrector which corrects for the spherical aberration and the off-axial coma of the imaging system. By design the novel hexapole corrector is free of intrinsic six-fold astigmatism $A_{5}$. The small negative fifth-order spherical aberration $\mathrm{C}_{5}$ is beneficial for positive phase contrast over a broad range of spatial frequencies. The $\mathrm{C}_{\mathrm{s}} / \mathrm{B}_{3}$-corrector provides means to correct for all parasitic axial aberrations up to fourth order. Hence, this corrector for the first time enables the realization of a fully aplanatic and fifth-order corrected CTEM.

The next-generation hexapole $\mathrm{C}_{\mathrm{s}}$-corrector CEOS B-COR for the conventional TEM has been integrated into the new Hitachi HF3300V TEM equipped with a cold field emission gun [4]. Due to the small intrinsic energy width and the good stability this aberration-corrected instrument provides an information limit better than $70 \mathrm{pm}$ at $300 \mathrm{kV}$. This has been verified with the Young's fringes method at a thin tungsten specimen (FIG. 1). The information limit illustrated by the projected fringe contrast (FIG. 2) can be attributed to a chromatic root mean square focus spread of $\operatorname{rms}\left(\mathrm{C}_{1}\right)=$ $1.46 \mathrm{~nm}$ due to the measured energy width of $\mathrm{dE}(\mathrm{fwhm})=0.45 \mathrm{eV}$ [4] and the total chromatic aberration of $\mathrm{C}_{\mathrm{c}}=2.28 \mathrm{~mm}$. Direct measurements of the total focus spread with the tilt method described in [2] actually indicate a slightly smaller value for the total focus spread of $\operatorname{rms}\left(\mathrm{C}_{1}\right)=$ $1.33+/-0.15 \mathrm{~nm}$.

The residual aberrations of the system at $300 \mathrm{kV}$ have been characterized with the diffractogram tableau method [5]. The axial aberrations up to fifth order have been calculated from the induced $\mathrm{C}_{1}$ and $A_{1}$ values for a tilt set with an outer tilt angle of $40 \mathrm{mrad}$ by least squares fitting [5]. To determine the coefficients of the generalized coma up to third order we recorded a tilt set with an outer tilt angle of 24 mrad. For each tilt position $C_{1}$ and $A_{1}$ have been evaluated at four off-axial $512 \times 512$ sub-regions of the $2 \mathrm{kx} 2 \mathrm{k}$ image. Both measurements were recorded with the same Nyquist frequency of $\mathrm{f}_{\mathrm{Ny}}=6.68 \mathrm{~nm}^{-1}$. From this data the linear variation of the lower-order axial aberrations over the field of view can be calculated with good precision. The results in TAB. 1 show that the azimuthal off-axial coma has been reduced from 0.7 to less than 0.1 while the parasitic off- 
axial two-fold astigmatism is below 0.01. All axial coefficients including the fourth order are corrected and the residual six-fold astigmatism is below $100 \mu \mathrm{m}$.

For the Hitachi HF3300V equipped with CEOS B-COR $\mathrm{C}_{\mathrm{s}}$-corrector the achievable state of correction, the measurement accuracy for the aberration assessment, and the information limit are sufficient to allow for almost optimum phase contrast conditions with an imaging aperture of 30 mrad at $300 \mathrm{kV}$ over a field of view of about $140 \mathrm{~nm}$.

References:

[1] M. Haider, et al. Advances in Imaging and Electron Physics 153, 43-119, 2008.

[2] M. Haider, et al. Microsc. Microanal. 16, 393-408, 2010.

[3] H. Müller, et al., Nucl. Instr. and Meth. A, doi: 10.1016/j.numa.2010.12.091, 2010.

[4] T. Sato, et al. Hitachi Rev. 57(3), 132-135, 2008.

[5] S. Uhlemann, et al. Ultramicroscopy 72, 109-119, 1998.

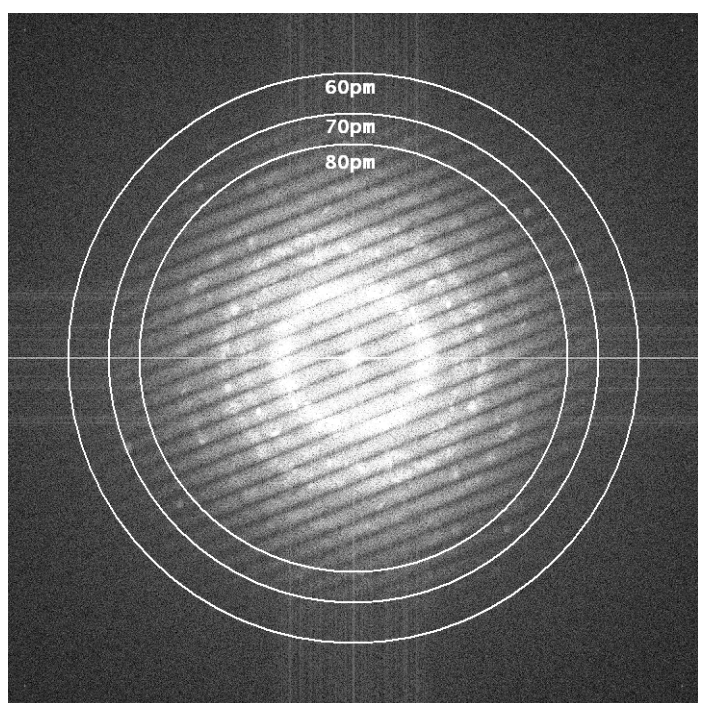

FIG. 1. Central region of a Young's fringe pattern from a thin tungsten specimen recorded at $300 \mathrm{kV}$ with $4 \mathrm{~s}$ illumination time. The Nyquist frequency in the original image is $38.3 \mathrm{~nm}^{-1 .}$

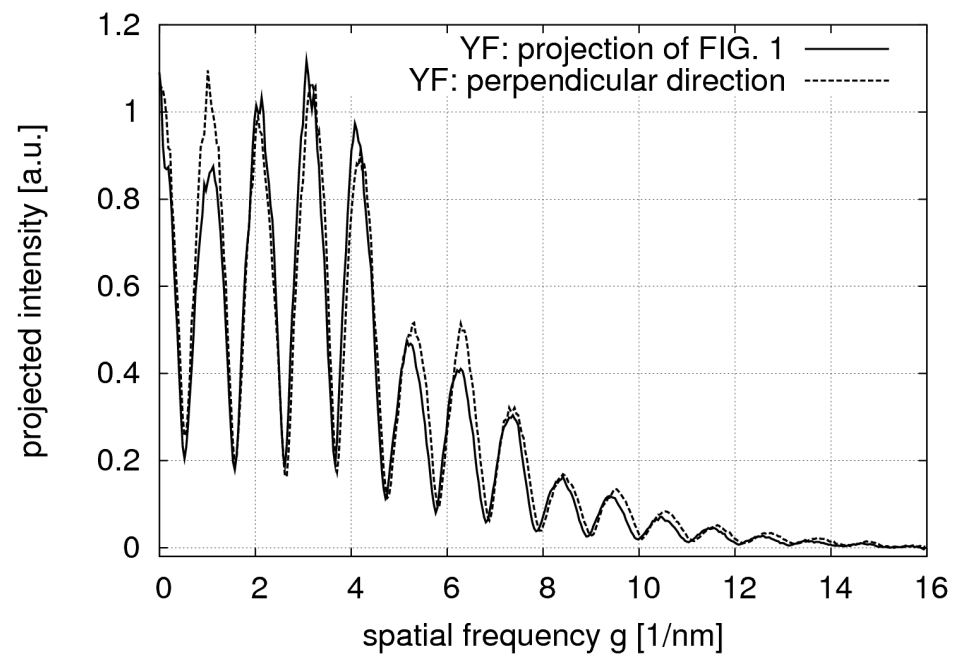

FIG. 2. Projected fringe contrast for two perpendicular directions from Young's fringe patterns. The specimen and the imaging conditions are the same as in FIG. 1.

\begin{tabular}{lll|lll|lll}
\hline coeff. & value & abs. err. & coeff. & value & abs. err. & coeff. & value & abs. err. \\
\hline$C_{1}$ & $-204 \mathrm{~nm}$ & $0.7 \mathrm{~nm}$ & $C_{1 \gamma}$ & $0.077 /-163^{\circ}$ & 0.002 & & & \\
$A_{1}$ & $0.5 \mathrm{~nm} /-79^{\circ}$ & $0.5 \mathrm{~nm}$ & $A_{1 \gamma}$ & $0.005 / 113^{\circ}$ & 0.004 & $A_{1 \bar{\gamma}}$ & $0.006 /-108^{\circ}$ & 0.004 \\
$B_{2}$ & $12 \mathrm{~nm} / 52^{\circ}$ & $11 \mathrm{~nm}$ & $B_{2 \gamma}$ & $0.03 /-130^{\circ}$ & 0.04 & $B_{2 \bar{\gamma}}$ & $0.07 /-51^{\circ}$ & 0.04 \\
$A_{2}$ & $14 \mathrm{~nm} / 128^{\circ}$ & $12 \mathrm{~nm}$ & $A_{2 \gamma}$ & $0.40 / 25^{\circ}$ & 0.07 & $A_{2 \bar{\gamma}}$ & $0.14 /-70^{\circ}$ & 0.01 \\
$C_{3}$ & $4.1 \mu \mathrm{m}$ & $0.8 \mu \mathrm{m}$ & $S_{3}$ & $49 \mathrm{~nm} / 55^{\circ}$ & $65 \mathrm{~nm}$ & $A_{3}$ & $100 \mathrm{~nm} /-99^{\circ}$ & $111 \mathrm{~nm}$ \\
$B_{4}$ & $9.3 \mu \mathrm{m} / 170^{\circ}$ & $2.6 \mu \mathrm{m}$ & $D_{4}$ & $5.0 \mu \mathrm{m} / 20^{\circ}$ & $1.0 \mu \mathrm{m}$ & $A_{4}$ & $5.3 \mu \mathrm{m} /-78^{\circ}$ & $2.2 \mu \mathrm{m}$ \\
$C_{5}$ & $-3.2 \mathrm{~mm}$ & $0.3 \mathrm{~mm}$ & $A_{5}$ & $49 \mu \mathrm{m} / 30^{\circ}$ & $46 \mu \mathrm{m}$ & & & \\
\hline
\end{tabular}

TAB. 1. Residual axial and off-axial aberration coefficients measured at $300 \mathrm{kV}$ from a tilt tableau with $40 \mathrm{mrad}$ (axial) and $24 \mathrm{mrad}$ (off-axial) aberrations. The absolute error is the radius of the $95 \%$ quantile estimated from the residuals of the least squares fit. 